\title{
GAIA Failure to Thrive Level of Diagnostic Certainty
}

National Cancer Institute

\section{Source}

National Cancer Institute. GAIA Failure to Thrive Level of Diagnostic Certainty. NCI

Thesaurus. Code C128673.

A classification of maternal and fetal outcomes relating to failure to thrive, developed by the Global Alignment of Immunization safety Assessment in pregnancy, based on the extent to which the diagnosis has been confirmed. 